\title{
6 Security, data rights and governance
}

New digital tools, such as digital biomarkers, can capture an unprecedented amount of information about users, including finegrained behavioral and physiological states. Many of these tools are non-invasive and collect data passively, which is certainly more convenient but also runs the risk that people do not understand how much of their digital footprint is being collected or shared. ${ }^{1}$ Recently granted patents include a shopping cart that monitors your heart rate and Alexa's new ability to apparently diagnose your cough. ${ }^{2}$ Data collected from such technologies could be used by a doctor to make a clinical decision or by an insurer to approve or deny a claim. ${ }^{1}$ Society needs to decide how to create systems that will deliver real benefits while protecting citizen privacy and safety. ${ }^{3}$

For example, there is a lot of excitement in the healthcare community about using these tools in postmarket monitoring, or surveillance, to track metrics like safety monitoring and efficacy. Although many of these surveillance techniques in healthcare are still in the early stages, security researchers in the tech world are understandably cautious. Put simply: personalized medicine holds great promise for humanity, but it is not possible to have personalized medicine without some amount of 'surveillance' - indeed, they go hand in hand. As de-identification gets more difficult with the vast amount of data generated for an individual, it is critical to understand, for an entity getting access to our data, who, what and when? ${ }^{4}$ Health insurers and data brokers have been vacuuming up personal details on individuals, to create predictions on health costs based on race, marital status, whether you pay your bills on time or even buy plus-size clothing. ${ }^{5}$ Similar data have been used to create 'health risk scores' for the opioid crisis, determining who gets access to what types of care. ${ }^{6}$ Many biases may not come to light until after more rigorous testing; for instance, researchers have found that Fitbits and other wearables may not accurately track heart rates in people of color. ${ }^{7}$ The biases in these types of algorithms are starting to become better 
documented, exacerbating health disparities - and yet our society lacks clear regulatory interventions or punishment for misuse. ${ }^{8,9}$

We have all heard about the 'Internet of Things' (IoT). What is coming next is the 'Internet of Bodies' (IoB) - a network of smart devices that are attached to or inside our bodies, as defined by Professor Andrea Matwyshyn. ${ }^{10}$ Most of the digital tools we have discussed fit within the IoB paradigm. Matwyshyn argues that using the human body as a technology platform raises a number of legal and policy questions for which regulators and judges need to prepare.

Our healthcare system has strong protections for how to store and share a patient's biological specimens, such as a blood or genomic sample - but what about our digital specimens? With the increase in biometric surveillance from these tools, data rights and governance - who gets access to what data and when - become critical. ${ }^{3}$

End user license agreements and terms of service, which gain consent one time on sign-up, are not sufficient as a method to actually inform a person about how their health data - in the form of a digital specimen - will be protected. ${ }^{11}$ Our society needs better social contracts with tech platforms that have accessible and meaningful informed consent processes baked into the product itself and can be tailored to adapt as user preferences change over time (Figure 6.1).

As the field of digital medicine, and indeed medicine as a whole, advances a process for the creation and promotion of policies, we need standards to ensure that people are protected from known and unknown harms due to myriad computational and human factors, including both failures of knowledge and failures of intent on the part of developers.

\section{Security and compliance}

Any organization that works with personally identifiable information, personal health information and direct access to patients is at a high risk for cyber threats. Today, with the rise of connected products, even a few vulnerable lines of code can have a profound impact on human life. Healthcare has seen a proliferation of vulnerabilities, particularly in connected technologies, many of which are life-critical: for example, Johnson \& Johnson's insulin pumps, St Jude Medical's implantable cardiac devices, and the WannaCry ransomware attack, which infected 200000 computers, many of which were part of a critical hospital 


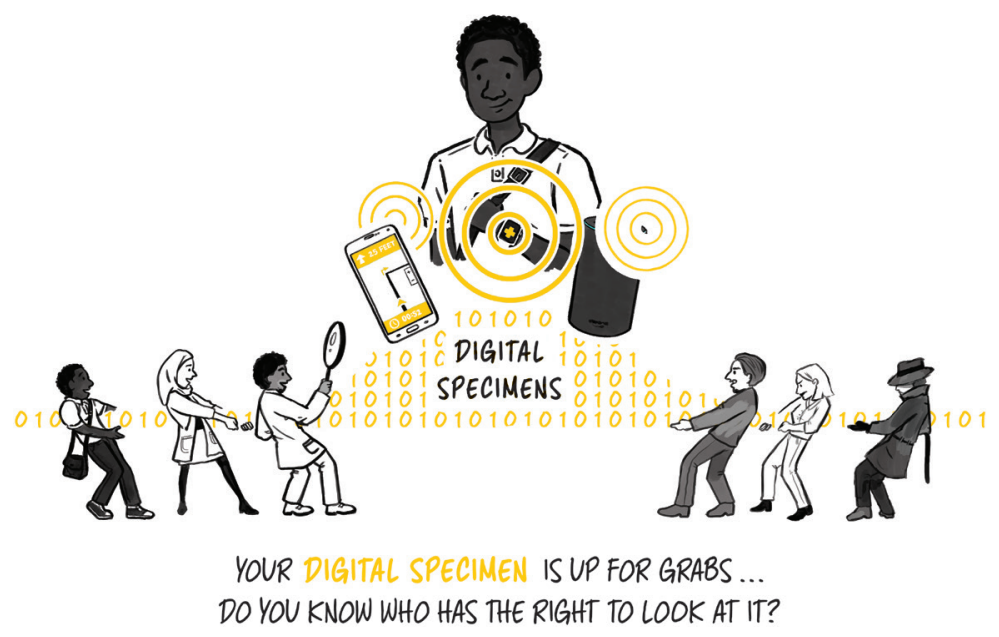

Figure 6.1 Digital specimens and social contracts. Our healthcare system has strong protections for patients' biological specimens, such as blood samples, but what about our 'digital specimens'? With the increase in biometric surveillance from these tools, data rights and governance - who gets access to what data and when - becomes critical.

infrastructure, across 150 countries. ${ }^{12-14}$ Vulnerabilities in connected technologies can be exposed by either black hat or white hat hackers.

- Black hat refers to a style of breaking into networks for personal or financial gain, often illegally without the owner's permission.

- White hat hackers, also referred to as security researchers, perform a style of ethical hacking on mission-critical networks and will employ the policies of coordinated disclosure to the network owner if vulnerabilities are found..$^{15}$

\section{Risk prioritization protocols}

A number of organizations have created protocols to prioritize risk levels of known vulnerabilities. For instance, MITRE, a non-profit that operates research and development centers sponsored by the federal government, created the Common Vulnerabilities and Exposures (CVE) program, to identify and catalog vulnerabilities in software or firmware into a free 'dictionary' for organizations to improve their security. ${ }^{16}$ 
Major agencies that are addressing healthcare cybersecurity include the National Institute of Standards and Technology, which has published a number of well-documented methods for quantitatively and qualitatively assessing cyber risks, and the US Food and Drug Administration (FDA), which has released a number of both premarket and postmarket guidances on cybersecurity best practices.

\section{Implementing protection policies}

Researchers and developers should not count on others to implement critical basic protections; they should have knowledge of their organization's policies and important contacts, such as the chief information security officer (CISO). For those embarking on this journey, check out $A$ cybersecurity primer for translational research; ${ }^{17}$ Box 6.1, for example, comes from this primer and elucidates the concepts of security and compliance, which are often confusing concepts for newcomers to the field.

\section{BOX 6.1}

\section{Security and compliance}

- Security is the application of protections and management of risk posed by cyber threats.

- Relates to how the technologies are updated, assessed and used.

- Compliance is typically a top-down mandate based on federal guidelines or law, whereas security is often managed bottom-up and is decentralized in most organizations.

- Typically relates to documentation (e.g. for the Health Insurance Portability and Accountability Act [HIPAA])

From Perakslis and Stanley, 2016. ${ }^{17}$

\section{Software bill of materials}

Most modern software is not written completely from scratch and includes common, off-the-shelf components, modules and libraries from both open-source and commercial teams. A tool to help manage potential vulnerabilities is called a 'software bill of materials' (SBOM), which is analogous to an ingredients list on food packaging and contains all the components in a given piece of software. The FDA 
has been investing more time and guidance around sharing SBOMs in both pre- and postmarket settings, and so have medical device makers like Philips and Siemens, and healthcare providers like NY Presbyterian and the Mayo Clinic. ${ }^{18-20}$

\section{The Hippocratic Oath for Connected Medical Devices}

As monitoring and surveillance tools become mainstream, it is critical to have secure and ethical checks and balances. For example, on graduation from medical school, soon-to-be physicians take the Hippocratic Oath, a symbolic promise to provide care in the best interest of patients. As connected tools increasingly augment clinicians, a critical question emerges: should the manufacturers and adopters of these connected technologies be governed by the symbolic spirit of the Hippocratic Oath ${ }^{21}$

Inspired by this idea, a number of security researchers from I Am The Cavalry, a grassroots organization with ties to DEF CON, an underground hacking conference, drafted 'The Hippocratic Oath for Connected Medical Devices' (HOCMD). ${ }^{22}$ This outlines a number of security and ethical principles, including 'secure by design' and 'resilience and containment'. ${ }^{21}$

While the FDA has not called out the HOCMD by name, in the 3 years since it was published, the FDA has incorporated elements from the five principles across its pre- and postmarket cybersecurity guidelines. ${ }^{23,24}$ The FDA has supported further collaboration between security researchers and connected device manufacturers through the agency-led \#WeHeartHackers initiative, which launched in early 2019. ${ }^{25}$

In August 2019, over ten medical device manufacturers, including Abbott, Thermo Fischer Scientific, Medtronic, Becton Dickinson, Philips and Siemens, convened at the Biohacking Village at DEF CON with the FDA in attendance. The manufacturers brought over 40 medical devices for white hat hackers to test and attack. The Mayo Clinic set up a model hospital environment where security researchers were able to be in an immersive hospital setting and executed a number of security challenges simulating a real environment. These types of collaborations between regulators, industry and researchers build stronger and more resilient systems for patients and the public health system at large. 


\section{Other initiatives}

Many government agencies support initiatives to improve security for medical connected technologies and healthcare delivery organizations. For instance, Health and Human Services (HHS) sponsors the Healthcare and Public Health Sector Coordinating Council (HSCC) joint Cybersecurity Working Group (CWG). The mission of the HSCC CWG is to collaborate with HHS and other federal agencies by crafting and promoting the adoption of recommendations and guidance for policy, regulatory and market-driven strategies to facilitate collective mitigation of cybersecurity threats to the sector that affect patient safety, security and privacy and consequently national confidence in the healthcare system. ${ }^{26}$

\section{Key points - security, data rights and governance}

- As advances in technology enable digital tools to gather ever larger amounts of high-resolution personal health information, core principles of medical and research ethics must be integrated at every step, beginning in the design phase.

- The line between 'personalization' and 'surveillance' is thin. As a result, and given the near impossibility to 'de-identify' mass amounts of data, it becomes more and more important to know who has access to sensitive data and under what conditions.

- Common practices in the consumer technology industry for obtaining agreement to corporate terms of service, including privacy policies, are not sufficient or appropriate for obtaining informed consent from users, be they patients receiving care or participants in health research. (When was the last time you read an end user license agreement for a website or app you use?)

- The field of digital medicine must develop innovative ways of ensuring that the values of respect, privacy and trust are not lost in the pursuit of better data. It is critical to ensure that the technologies are worthy of the trust we place in them. 


\section{References}

1. Allen M. You snooze, you lose: insurers make the old adage literally true. Pro Publica, 2018. www. propublica.org/article/you-snoozeyou-lose-insurers-make-the-oldadage-literally-true, last accessed 31 July 2019.

2. Mehta I. Amazon's new patent will allow Alexa to detect a cough or a cold. TNW, 2018. https:// thenextweb.com/artificialintelligence/2018/10/15/amazonsnew-patent-will-allow-alexa-todetect-your-illness/, last accessed 31 July 2019.

3. Perakslis E. Protecting patient privacy and security while exploiting utility of next generation digital heath wearables. BMJ Opinion, 2019. https://blogs.bmj.com/ bmj/2019/01/18/protecting-patientprivacy-and-security-whileexploiting-the-utility-of-nextgeneration-digital-health-wearables/, last accessed 31 July 2019.

4. Berinato $S$. There is no such thing as anonymous data. Harvard Business Review, 2015. https://hbr. org/2015/02/theres-no-such-thing-asanonymous-data, last accessed 31 July 2019.

5. Allen M. Health insurers are vacuuming up details about youand it could raise your rates. ProPublica, 2018. www.propublica. org/article/health-insurers-arevacuuming-up-details-about-youand-it-could-raise-your-rates, last accessed 31 July 2019.
6. Ravindranath M. How your health information is sold and turned into risk scores. Politico, 2019. www. politico.com/story/2019/02/03/ health-risk-scores-opioid-abuse1139978 ?mc_cid=e83d0f0d3e\&mc eid $=4 d 57$ c573ca, last accessed 31 July 2019.

7. Hailu R. Fitbits and other wearables may not accurately track heart rates in people of color. STAT, 2019. www.statnews. com/2019/07/24/fitbit-accuracydark-skin/, last accessed 24 September 2019.

8. Khullar DA. AI could worsen health disparities. New York Times, 2019. www.nytimes.com/2019/01/ 31/opinion/ai-bias-healthcare.html [paywall], last accessed 31 July 2019.

9. Coravos A, Chen I, Gordhandas A, Stern AD. We should treat algorithms like prescription drugs. Quartz, 2019. https://qz. com/1540594/treating-algorithmslike-prescription-drugs-could-reduceai-bias/, last accessed 31 July 2019.

10. Matwyshyn AM. The internet of bodies (January 1, 2018). William \& Mary Law Review 2019;61. Available from SSRN: https://ssrn.com/ abstract $=3452891$.

11. Perakslis E, Coravos A. Is health-care data the new blood? Lancet Digital Health 2019;1:PE8-9.

12. Rockoff JD. J\&J warns insulin pump vulnerable to cyber hacking. Wall Street Journal, 2016. www.wsj. com/articles/j-j-warns-insulinpump-vulnerable-to-cyber-hacking1475610989, last accessed 31 July 2019. 
13. Larson S. FDA confirms that St. Jude's cardiac devices can be hacked. CNN Business, 2017. https://money. cnn.com/2017/01/09/technology/ fda-st-jude-cardiac-hack/index.html, last accessed 31 July 2019.

14. Brewster T. Medical devices hit by ransomware for the first time in US hospitals. Forbes, 2017. www.forbes. com/sites/thomasbrewster/ 2017/05/17/wannacry-ransomwarehit-real-medical-devices/\#dad $2 \mathrm{~b} 18425 \mathrm{cf}$, last accessed 31 July 2019.

15. I Am The Cavalry. I Am The Cavalry Position on Disclosure, 2018. www.iamthecavalry.org/about/ disclosure/, last accessed 31 July 2019.

16. Common Vulnerabilities and Exposures. https://cve.mitre.org, last accessed 31 July 2019.

17. Perakslis ED, Stanley M. A cybersecurity primer for translational research. Sci Transl Med 2016;8:322.

18. Philips. Position Paper: Committed to Proactively Addressing Our Customers Security and Privacy Concerns, 2018. www.philips.com/cdam/corporate/ newscenter/global/standard/ resources/healthcare/2018/ cybersecurity/Cybersecurity_ position_paper.pdf, last accessed 31 July 2019.

19. Aske J, Jacobson J. NTIA Software Component Transparency: Healthcare Proof of Concept at a Glance, 2018. www.ntia.doc.gov/files/ntia/ publications/healthcare_wg_-proof_of_concept_overview.pdf, last accessed 31 July 2019.
20. Mayo Clinic. Medical and Research Device Risk Assessment Vendor Packet Instructions, 2019. www.mayoclinic. org/documents/medical-devicevendor-instructions/doc-20389647, last accessed 31 July 2019.

21. Woods B, Coravos A, Corman JD. The case for a Hippocratic Oath for Connected Medical Devices: viewpoint. J Med Internet Res 2019; 21:e12568.

\section{I Am The Cavalry. Hippocratic} Oath for Connected Medical Devices. www.iamthecavalry.org/domains/ medical/oath/, last accessed 31 July 2019.

\section{US Food and Drug} Administration. Content of Premarket Submissions for Management of Cybersecurity in Medical Devices, 2018. www.fda.gov/regulatory-information/ search-fda-guidance-documents/ content-premarket-submissionsmanagement-cybersecurity-medicaldevices, last accessed 31 July 2019.

24. US Food and Drug Administration. Postmarket Management of Cybersecurity in Medical Devices, 2016. www.fda.gov/ regulatory-information/search-fdaguidance-documents/postmarketmanagement-cybersecurity-medicaldevices, last accessed 31 July 2019.

25. \#WeHeartHackers. http:// wehearthackers.org, last accessed 31 July 2019.

26. Healthcare and Public Health Sector Critical Infrastructure Security and Resilience Partnership. https:// healthsectorcouncil.org, last accessed 31 July 2019. 